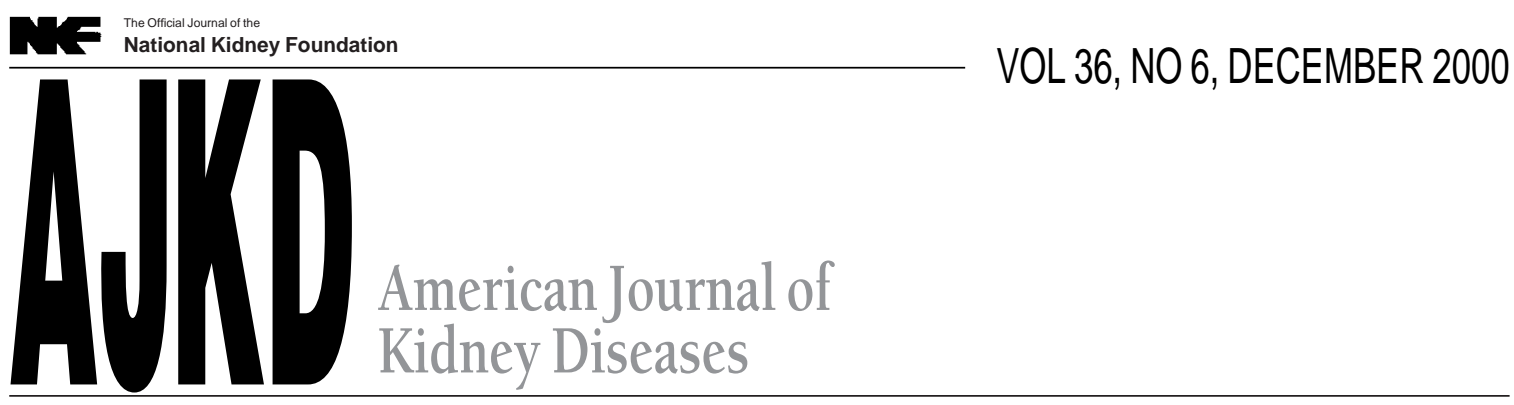

IN-DEPTH REVIEW

\title{
Cholesterol Crystal Embolism: A Recognizable Cause of Renal Disease
}

\author{
Francesco Scolari, MD, Regina Tardanico, MD, Roberta Zani, MD, Alessandra Pola, MD, \\ Battista Fabio Viola, MD, Ezio Movilli, MD, and Rosario Maiorca, MD
}

- Cholesterol crystal embolism, sometimes separately designated atheroembolism, is an increasing and still underdiagnosed cause of renal dysfunction antemortem in elderly patients. Renal cholesterol crystal embolization, also known as atheroembolic renal disease, is caused by showers of cholesterol crystals from an atherosclerotic aorta that occlude small renal arteries. Although cholesterol crystal embolization can occur spontaneously, it is increasingly recognized as an iatrogenic complication from an invasive vascular procedure, such as manipulation of the aorta during angiography or vascular surgery, and after anticoagulant and fibrinolytic therapy. Cholesterol crystal embolism may give rise to different degrees of renal impairment. Some patients show only a moderate loss of renal function; in others, severe renal failure requiring dialysis ensues. An acute scenario with abrupt and sudden onset of renal failure may be observed. More frequently, a progressive loss of renal function occurs over weeks. A third clinical form of renal atheroemboli has been described, presenting as chronic, stable, and asymptomatic renal insufficiency. The renal outcome may be variable; some patients deteriorate or remain on dialysis, some improve, and some remain with chronic renal impairment. In addition to the kidneys, atheroembolization may involve the skin, gastrointestinal system, and central nervous system. Renal atheroembolic disease is a difficult and controversial diagnosis for the protean extrarenal manifestations of the disease. In the past, the diagnosis was often made postmortem. However, in the last decade, awareness of atheroembolic renal disease has improved, enabling us to make a correct premortem diagnosis in a number of patients. Correct diagnosis requires the clinician to be alert to the possibility. The typical patient is a white man aged older than 60 years with a baseline history of hypertension, smoking, and arterial disease. The presence of a classic triad characterized by a precipitating event, acute or subacute renal failure, and peripheral cholesterol crystal embolization strongly suggests the diagnosis. The confirmatory diagnosis can be made by means of biopsy of the target organs, including kidneys, skin, and the gastrointestinal system. Thus, Cinderella and her shoe now can be well matched during life. Patients with renal atheroemboli have a dismal outlook. A specific treatment is lacking. However, it is an important diagnosis to make because it may save the patient from inappropriate treatment. Finally, recent data suggest that an aggressive therapeutic approach with patient-tailored supportive measures may be associated with a favorable clinical outcome.

(C) 2000 by the National Kidney Foundation, Inc.

INDEX WORDS: Atherosclerosis; cholesterol crystals; renal atheroembolic disease.

$\mathbf{C}$ HOLESTEROL crystal embolism, sometimes separately designated atheroembolism, is a multisystemic disorder that generally affects many organs. It is characterized by the occlusion of small arteries by cholesterol crystal emboli deriving from eroded atherosclerotic plaques of the aorta or large feeder arteries. ${ }^{1-6}$ It is often stated that cholesterol crystal embolism was first described more than 100 years ago by the German pathologist, Panum. ${ }^{7}$ However, the first reliable description of this disease was made
From the Division and Chair of Nephrology and Department and Chair of Pathology, Spedali Civili and University, Brescia, Italy.

Received November 1, 1999; accepted in revised form May 12, 2000.

Address reprint requests to Francesco Scolari, MD, Division of Nephrology, Spedali Civili, P le Spedali Civili, 1, 25125 Brescia, Italy.E-mail: fscolar@tin.it

(C) 2000 by the National Kidney Foundation, Inc.

0272-6386/00/3606-0001\$3.00/0

doi:10.1053/ajkd.2000.19809 
by Flory ${ }^{8}$ in 1945 . Subsequently, it evolved slowly from a pathological curiosity to a clinical entity. ${ }^{1-3}$ The proximity of the kidneys to the abdominal aorta and the large renal blood supply make the kidney a frequent target organ for atheroemboli.

However, cholesterol crystal embolization has been frequently overlooked as a cause of renal dysfunction during life. In the past, the diagnosis of renal cholesterol crystal embolization, also designated atheroembolic renal disease, often was made postmortem. ${ }^{1-3}$ For this reason, the disorder has been called the Cinderella of nephrology: Cinderella (the patient and clinical findings) and her shoe (the final diagnosis of atheroembolic renal disease) are not usually matched in life. ${ }^{9,10}$ However, in the last few years, large retrospective clinical studies have increased our understanding of atheroembolic renal disease..$^{11-14}$ Better knowledge of the disease now permits a correct premortem diagnosis in a significant number of cases: Cinderella and her shoe now can be well matched during life.

The purpose of this review is to discuss the pathogenesis and incidence of atheroembolic renal disease, its clinical and pathological findings, and the potential for a correct premortem diagnosis.

\section{PATHOGENESIS}

\section{The Aorta in Atheroembolic Renal Disease}

The hypothesis that cholesterol crystal embolism may originate from a diseased atherosclerotic aorta was first made by Flory ${ }^{8}$ in 1945. Among 267 consecutive autopsies, he observed nine instances of cholesterol crystal embolism: none in 63 cases in which aortic plaque ulceration was absent, two instances in 147 cases (1\%) with moderate aortic plaque erosion, and seven instances in 57 cases (12\%) with severe aortic plaque ulceration. Since then, other investigators have confirmed the strict correlation of cholesterol crystal embolism with the severity of atherosclerotic aortic plaque erosion. In patients who died with cholesterol crystal emboli syndrome, autopsy findings usually showed the internal lining of the aorta as irregularly ulcerated and extensively covered by soft, friable, thrombotic material; in many areas, the soft, friable, yellow contents of the inner core of the atherosclerotic
Table 1. Atheroembolic Renal Disease: Risk Factors and Prior Medical Problems

\begin{tabular}{ll}
\hline Risk Factors & \multicolumn{1}{c}{ Prior Medical Problems } \\
\hline Male sex & Ischemic cardiovascular disease \\
Age $>60$ y & Cerebrovascular disease \\
White race & Abdominal aortic aneurysm \\
Hypertension & Ischemic nephropathy \\
Tobacco use & Peripheral vascular disease \\
Diabetes mellitus & \\
\hline
\end{tabular}

lesion communicated with the aortic lumen. ${ }^{15-17}$ Thus, available data suggest that severe ulcerated atherosclerosis is primarily responsible for the atheroembolic renal disease. This is further supported by the finding that risk factors for the development of atherosclerosis, such as older age, male sex, diabetes, hypertension, and cigarette smoking, are the same risk factors for developing atheroembolic renal disease (Table 1).

\section{Precipitating Factors}

The formation of a complicated lesion of atherosclerosis is a prerequisite for the development of cholesterol crystal emboli syndrome. In the past decade, our understanding of plaque biological characteristics has increased dramatically, and the concept of vulnerable atherosclerotic plaque has emerged. ${ }^{18}$ Atherosclerotic plaques consist of a fibrous cap, under which are macrophages, necrotic debris, and cholesterol crystals. Plaques with a large extracellular lipid-rich core and a thin fibrous cap appear to be most vulnerable to rupture. Mechanical and hemodynamic stresses may fatigue and weaken the fibrous cap, which ultimately may rupture, releasing the underlying extracellular cholesterol-rich matrix. Atheroembolization may occur spontaneously or after aortic wall trauma, including vascular surgery and angiographic procedures. Other etiologic factors include anticoagulation and thrombolysis. A number of patients may experience multiple triggering factors, such as a combination of anticoagulation, angiographic procedures, and vascular surgery. ${ }^{1-6,11-14,19,20}$

Vascular surgery procedures known to precipitate cholesterol crystal embolism include abdominal aortic aneurysm resection and graft, aortoiliac and aortofemoral bypass, carotid endarterectomy, cardiac surgery, and renal artery 
grafts. Vascular surgery may disrupt plaques when incisions are made through the walls of the vessel or when the vessel is cross-clamped or manipulated during surgery. ${ }^{1-6}$ The occurrence of atheroemboli may be observed after radiological instrumentation of the aorta, such as aortography, coronary angiography, cardiac catheterization, and percutaneous transluminal angioplasty of the coronary bed, renal arteries, or other arterial systems. The proposed mechanism is mechanical trauma. Catheter manipulations scrape the walls of the aorta, disrupting atherosclerotic plaques and exposing the soft, cholesterol-laden core of the plaque to the arterial circulation. ${ }^{1-6}$

Coronary angiography is considered the most common arteriographic procedure inciting cholesterol crystal embolism. ${ }^{1-6,11-14}$ Because atherosclerotic plaques are most common in the abdominal aorta, some investigators suggest using the brachial approach when there is evidence of severe aortic atherosclerosis. However, a recent prospective study of 1,579 patients undergoing coronary angioplasty did not show significant differences between the brachial and femoral approaches. ${ }^{21}$ However, an important limitation of this study was the low number of events, because only one patient developed atheroembolization. Thus, whether the use of the brachial approach is associated with a lower incidence of atheroemboli is still a matter of controversy.

The third precipitating factor described is anticoagulant treatment, including both heparin and oral anticoagulants (warfarin [Coumadin; DuPont Pharma, Wilmington, DE]). These agents may prevent the formation of a protective thrombus overlying an ulcerated plaque or may initiate the disruption of a complex plaque by causing hemorrhage into it. ${ }^{1-6}$ With the advent of thrombolytic therapy for the treatment of myocardial infarction, there have been several case reports in which the precipitating factor has been the use of these agents. ${ }^{19}$ The effect of thrombolytic therapy is to actively lyse thrombi, including those covering atherosclerotic plaques. Despite this, atheroembolism is a complication usually ignored in the megatrials of thrombolytic therapy of acute myocardial infarction. ${ }^{22}$ In 1995, Blankenship et $\mathrm{al}^{23}$ in a prospective study, did not show a significantly greater prevalence of cholesterol crystal embolism in patients administered thrombolytic therapy than in those treated conservatively. However, the study had a small sample size (60 patients). In addition, the patients underwent cardiac catheterization before surgery, which can itself promote atheroembolization. Thus, larger studies are needed to find or exclude a relation between thrombolytic therapy and cholesterol crystal embolization.

In early reports, a greater percentage of patients with atheroembolic renal disease (range, $40 \%$ to $69 \%$ ) showed a spontaneous form of disease.$^{1-3}$ However, in more recent reports, cholesterol crystal embolization was much more frequently associated with precipitating factors. ${ }^{11-14}$ This may reflect the increased use of arterial invasive procedures and the widespread use of anticoagulation and thrombolysis.

Our own data confirm the growing role of invasive procedures and anticoagulation as a triggering factor. From 1989 to 1999, a total of 52 patients ( 44 men, 8 women; average age, 68 years) with a diagnosis of atheroembolic renal disease were collected at our institution. The disease was spontaneous in 11 patients $(21 \%)$, and a triggering factor was identified in 41 patients $(79 \%)$. Aortic surgery, consisting of aneurysm resection, was the precipitating factor in 8 patients $(15 \%)$. Twenty-six patients $(50 \%)$ developed renal atheroembolism after angiography. Aortic or coronary angiography through the femoral artery was the sole triggering factor in 16 patients. Angiography was followed by an angioplastic procedure in 6 patients (4 patients, renal artery; 2 patients, coronary artery); in 2 of these patients, an interventional radiological procedure was completed by stenting. In 4 more patients, angiography was followed by exposure to an additional inciting factor: aortic surgery in 3 patients and thrombolytic therapy in 1 patient. Finally, in 11 patients $(21 \%)$, the triggering factor was anticoagulant treatment, including thrombolytic therapy, calcic heparin, and oral anticoagulants.

\section{FATE OF ATHEROEMBOLI}

Once in the circulation, cholesterol crystal emboli lodge in small arteries 150 to $200 \mu \mathrm{m}$ in diameter. The lodgment of cholesterol clefts is followed by an inflammatory reaction, often of a granulomatous type, involving macrophage and 
giant-cell infiltration. The foreign-body inflammatory reaction leads to an endothelial reaction, causing intimal proliferation and intravascular fibrosis of the affected vessels. The entire process of cholesterol crystal embolism and vascular reaction results in narrowing or obliteration of the lumen. ${ }^{1,17,24}$ These pathological changes result in tissue effects distal to the cholesterol crystal emboli and include ischemia and, rarely, infarction, depending on the extent of organ involvement.

On the basis of ultrastructural observation of cholesterol crystal emboli in renal biopsies, Jones and Iannacone ${ }^{25}$ divided the changes found into three phases. The early phase consists of fresh crystal emboli causing endothelial injury and an early histiocytic response. The intermediate phase is characterized by a giant-cell reaction and intimal proliferation. The late phase shows the encasement of the crystals by histiocytes, more intimal proliferation, and fibrous tissue formation.

The sequential changes in the lesions in human atheroembolism closely resemble those of cholesterol crystal emboli disease in experimental animals. Flory ${ }^{8}$ first attempted to reproduce cholesterol crystal emboli in rabbits. Cholesterol crystal embolic lesions were identified in the pulmonary vessels. At 24 hours, there was a marked leukocytic response surrounding the arterial lesion; at 7 days, sequestration of the cholesterol crystals by foreign-body giant cells and scar was found. Snyder and Shapiro ${ }^{26}$ amplified these observations. Cholesterol clefts could be identified in the small pulmonary arteries of rabbits 1 day after the injection. By 3 days, there was panarteritis with numerous eosinophils; the intima was hyperplastic, and foreign-body giant cells were also found around the crystals. By 12 days, intimal fibrosis and sequestration of the crystal by foreign-body giant cells occurred. No further changes were observed at 160 days. This experimental work showed the persistence of cholesterol crystal emboli for long periods, confirming that cholesterol crystals, insoluble in body fluids and not removable by phagocytosis, may act as a permanent foreign body. In conformity with these findings, Gore et $\mathrm{al}^{27}$ found that the cholesterol crystals may be shown in rats 9 months after the acute event, confirming that they remain unadsorbed.

Finally, the time course of the renal tissue reaction was studied by Warren and Vales, ${ }^{28}$ who injected atherosclerotic material into rabbit renal arteries. In the first days, an occlusive thrombus was noted that incorporated cholesterol crystals, debris, platelet aggregates, and fibrin. This was followed by partial temporary resolution of the thrombus, with partial recanalization of the lumen. In a final late phase, identified from 5 days to 1 month, extensive fibrosis around cholesterol crystals was noted. Thus, experimental and human studies provide a picture of an evolving vascular lesion. The rapidity of the fibrotic process is a point deserving consideration and seems to be caused by the intrinsic properties of atherosclerotic material, which is highly thrombogenic and may also trigger complement activation.

\section{EPIDEMIOLOGICAL CHARACTERISTICS}

The syndrome of renal cholesterol crystal embolism usually affects elderly men with a history of diffuse atherosclerosis. ${ }^{1-3}$ In the largest reports, mean age varied from 66 to 70 years (range, 45 to 84 years). ${ }^{2,3,11-14}$ The disease is more frequently diagnosed in men than women, ${ }^{1-3,11-14}$ which may be explained by a difference in the prevalence of atherosclerosis between these groups. ${ }^{2}$ A review of the literature regarding the racial incidence of atheroembolism suggests that it predominately affects whites. ${ }^{29}$ Because blacks appear to have an increased prevalence of atherosclerosis compared with whites, it has been suggested that this diagnosis may be underrecognized in patients with dark skin. ${ }^{2}$

The true incidence of atheroembolic renal disease is difficult to estimate. Recent studies showed that this entity is not a rare event in everyday clinical practice. Mayo and Swartz ${ }^{14}$ reviewed 402 consultation charts in a single institution, suggesting that the incidence of clinically detectable atheroembolism amounted to at least $4 \%$ of all inpatients examined. Belenfant et $\mathrm{al}^{12}$ reported that $3 \%$ of 2,102 patients admitted to their tertiary renal intensive care unit over a 12-year period were diagnosed as having renal atheroembolism. Our series includes 52 cases of atheroembolic renal disease identified at our institution over a 10-year period. The frequency of this diagnosis increased during the period, and in the 
Table 2. Incidence of Renal Atheroembolism

\begin{tabular}{|c|c|c|}
\hline Reference & Incidence (\%) & Population Under Study \\
\hline \multicolumn{3}{|l|}{ Autopsy studies } \\
\hline Kealy ${ }^{16}$ & 1 & Unselected series $(n=2,126)$ \\
\hline Cross $^{30}$ & 2.4 & Unselected series $(n=372)$ \\
\hline Moolenar and Lamers ${ }^{31}$ & 0.31 & Unselected series $(n=89,075)$ \\
\hline \multirow[t]{3}{*}{ Flory 8} & 12.3 & Severe aortic atherosclerosis $(n=57)$ \\
\hline & 1 & Moderate aortic atherosclerosis $(n=147)$ \\
\hline & 0 & No aortic atherosclerosis $(n=63)$ \\
\hline \multirow[t]{4}{*}{ Thurlbeck and Castleman ${ }^{15}$} & 77 & Aortic surgery $(n=22)$ \\
\hline & 31 & Nonoperated aneurysm $(n=42)$ \\
\hline & 15.8 & Severe atherosclerosis $(n=38)$ \\
\hline & 0 & Minimal atherosclerosis $(n=44)$ \\
\hline Gore and Collins 32 & 17.6 & Subjects $>60$ y $(n=34)$ \\
\hline Ramirez et al ${ }^{20}$ & 27 & Cardiac catheterization $(n=71)$ \\
\hline \multicolumn{3}{|l|}{ Kidney biopsy studies } \\
\hline Jones and lannacone ${ }^{25}$ & 1 & Consecutive biopsies $(n=755)$ \\
\hline $\mathrm{Lie}^{24}$ & 1 & Consecutive biopsies $(n=4,580)$ \\
\hline Preston et al ${ }^{33}$ & 3.4 & Subjects $\geq 65$ y $(n=334)$ \\
\hline \multirow[t]{2}{*}{ Stone and Fogo ${ }^{34}$} & 0.8 & Consecutive biopsies $(n=1,219)$ \\
\hline & 5.5 & Elderly subjects $(n=91)$ \\
\hline \multicolumn{3}{|l|}{ Angiographic studies } \\
\hline Drost et al ${ }^{35}$ & 0.15 & Cardiac catheterization $(\mathrm{n}=4,578)$ \\
\hline Colt et $\mathrm{al}^{5}$ & 0.18 & Cardiac catheterization $(n=3,733)$ \\
\hline Johnson et al ${ }^{21}$ & 0.06 & Coronary angioplasty $(n=1,579)$ \\
\hline Frock et al ${ }^{36}$ & 0.1 & Angiography $(n=14,998)$ \\
\hline
\end{tabular}

last 2 years, we estimate encountering cases of atheroembolism as frequently as once monthly.

However, these retrospective surveys do not allow incidence calculation of atheroembolic renal disease because patients with severe atheroembolic renal failure are usually overrepresented, representing the "tip of the iceberg." Estimates of renal cholesterol crystal embolism frequency are mainly derived from autopsy or renal biopsy examinations and case series of angiographic studies (Table 2). Retrospective autopsy or biopsy studies may exaggerate the frequency of the disease because they may detect subclinical cases. Conversely, the incidence of atheroembolic renal disease in clinical studies appears to be much less than that observed in autopsy and biopsy studies. However, in these studies, clinically significant atheroembolism has probably been underestimated because of shortterm follow-up. Among unselected series of autopsies, the frequency of atheroemboli is generally considered to be low, ranging from $0.31 \%$ to 2.4\%.$^{16,30,31}$ However, autopsy studies performed in selected populations, such as older subjects and patients who died after aortic surgery and aortography, have reported greater frequencies of cholesterol crystal embolism, varying from $12 \%$ to $77 \% .^{15,16,20,32}$

Systematic studies of the antemortem diagnosis of renal cholesterol crystal embolization in humans are very few. Jones and Iannacone ${ }^{25}$ and $\mathrm{Lie}^{24}$ found a $1 \%$ incidence of renal atheroemboli in series of 755 and 4,580 consecutive renal biopsies, respectively. However, when older subjects are considered, the prevalence is greater. In a survey of 334 renal biopsies performed on patients aged 65 years or older, Preston et $\mathrm{al}^{33}$ found 14 cases $(4.2 \%)$ of cholesterol crystal embolism. In 1992, Stone and Fogo ${ }^{34}$ found a prevalence of cholesterol crystal embolism of $0.8 \%$ in 1,219 consecutive renal biopsies; however, in 91 elderly patients, a prevalence of $5.5 \%$ was documented. ${ }^{34}$ Estimates of the incidence of renal atheroembolic disease after aortic catheterization are varied and appear to be much less than those observed in autopsy and biopsy studies. The four largest catheterization studies of the literature, involving 24,897 patients, cited an incidence less than $0.2 \%$ (range, $0.06 \%$ to $0.18 \%)^{5,21,35,36}$ However, it remains difficult to quantify the magnitude of clinically significant angiography-associated atheroembolism because 
the majority of the studies did not search the disease systematically, which can manifest weeks to months after the procedure.

\section{PATHOLOGICAL CHARACTERISTICS}

The histological features of renal cholesterol crystal embolization are highly characteristic. ${ }^{1-3,25}$ The hallmark of the condition is an occlusion of the lumen of small arteries by atherosclerotic material. Because the lipids are dissolved by the techniques used to prepare the tissue for histological examination, the cholesterol crystals may be identified by the presence of needle-shaped spaces, which appear empty in routine histological sections. Thus, clefts are found that remain after cholesterol has dissolved during fixation. Renal histological examination shows characteristic, biconvex, needle-shaped clefts in vessels between 50 and $200 \mu \mathrm{m}$ in diameter (arcuate and interlobular arteries). Some vessels are completely obliterated; others show a residual lumen. Rarely, the cholesterol crystals lodge in the afferent arteries and glomeruli. The clefts occasionally may be seen singly in a vessel; usually, they consist of clusters of elongated biconvex needle-shaped crystals lying parallel to one another. However, in frozen sections, the crystals are highly birefringent and give positive histochemical reactions for lipids. ${ }^{1-3}$ In acute lesions, the crystals are embedded in lipid-rich amorphous debris and blood clot; an inflammatory response characterized by polymorphonuclear leukocytes and eosinophils may be seen transiently. At later stages, foreign-body giant cells, endothelial proliferation, and fibrous tissue surrounding the crystals are found, leading to luminal obliteration. ${ }^{25}$ Occlusion of vessels 150 to $200 \mu \mathrm{m}$ in diameter can account for the changes in renal parenchyma. Numerous foci of patchy, irregular atrophy of the renal parenchyma and occasional areas of ischemic infarction may be seen. In the involved areas, glomeruli show various degrees of obsolescence or ischemic retraction of the tuft. Many tubules show atrophic changes from slight to severe; areas of acute tubular necrosis may also be present. The interstitium appears to be enlarged by diffuse fibrosis. ${ }^{1-3}$

Sporadically, other renal histological features have been described, such as necrotizing glomerulonephritis and crescenteric changes. ${ }^{2,3}$ In addi- tion to the changes caused by vascular occlusion by cholesterol crystals, other renal parenchymal changes related to associated underlying diseases may be found. Because the patients with atheroembolism usually have diffuse atherosclerosis and hypertension, varying degrees of nephroangiosclerotic lesions may be seen. ${ }^{37,38} \mathrm{~A}$ picture of glomerulosclerosis secondary to diabetes mellitus, which is considered a risk factor for the disease, may be superimposed. ${ }^{13}$ The findings on gross examination of the kidneys reflect the previously mentioned parenchymal changes. Both kidneys are usually reduced in size and have a rough granular surface with wedgeshaped vascular scars. Numerous patchy, yellowwhite, wedge-shaped, atrophic areas of ischemia are surrounded by zones of unaffected tissue. On gross inspection, only occasional areas of acute infarcts may be seen..$^{1-3}$ In addition to the kidney, atheroemboli have been found in many anatomic sites, including skin, muscles, spleen, gastrointestinal system, heart, brain, bone, prostate, bladder, penis, testes, and spinal cord. ${ }^{1-3}$

\section{CLINICAL FEATURES}

The clinical consequences of cholesterol crystal embolization vary considerably. Patients may be completely asymptomatic when the diagnosis is made coincidentally at renal biopsy, or they may present with a distinct clinical syndrome, ranging from a cyanotic toe to a multiorgan systemic disease that can mimic other systemic diseases $^{1-6,11-14}$ (Table 3). The distribution of endorgan damage depends on the anatomic location of the original atherosclerotic plaques and the extent of organ involvement. For instance, cholesterol crystal embolization from carotid artery plaques leads to clinical involvement confined to the retinal and cerebral circulation. However, when the source of the atheroemboli is in the thoracic aorta or, more commonly, the abdominal aorta, the central nervous system, visceral organs, and extremities may be involved. Only the lower extremities are involved if the site is below the renal arteries.

From a clinical point of view, cholesterol crystal embolism is a puzzling event, and atheroembolic renal disease is part of a multisystem disease. Moreover, individuals at risk for renal cholesterol crystal embolism commonly have other chronic illnesses causing renal failure, such 
Table 3. Clinical and Laboratory Features of Atheroembolic Renal Disease in the Five Largest Case Series

\begin{tabular}{|c|c|c|c|c|c|}
\hline & \multicolumn{5}{|c|}{ Reference } \\
\hline & Fine et $\mathrm{al}^{2}$ & Lye et $\mathrm{al}^{3}$ & Thadhani et al ${ }^{11}$ & Belenfant et al ${ }^{12}$ & Scolari et al ${ }^{*}$ \\
\hline No. of patients & 221 & 129 & 52 & 67 & 52 \\
\hline Spontaneous form (\%) & 69 & 40 & 0 & 4 & 21 \\
\hline latrogenic form† (\%) & 31 & 60 & 100 & 96 & 79 \\
\hline Radiology procedure (\%) & 18 & 43 & 96 & 85 & 50 \\
\hline Cardiovascular surgery (\%) & 9 & 5 & 41 & 36 & 15 \\
\hline Anticoagulation (\%) & 14 & 13 & 37 & 76 & 21 \\
\hline Skin lesions (\%) & 35 & 43 & 50 & 90 & 96 \\
\hline Gl involvement (\%) & 10 & 10 & 29 & 33 & 8 \\
\hline CNS involvement (\%) & - & 12 & 23 & 4 & 8 \\
\hline Retinal emboli (\%) & 6 & 10 & 25 & 22 & 8 \\
\hline Eosinophilia (\%) & 73 & 71 & 22 & 59 & 62 \\
\hline \multicolumn{6}{|l|}{ Outcome } \\
\hline CRF requiring dialysis (\%) & 28 & 40 & 44 & 61 & 35 \\
\hline Recovery from dialysis dependence (\%) & - & 21 & 26 & 32 & 27 \\
\hline 1-Year mortality rate (\%) & 81 & 64 & 87 & 23 & 31 \\
\hline
\end{tabular}

Abbreviations: GI, gastrointestinal; CNS, central nervous system.

* Current study.

† Some patients experienced more than one triggering factor.

as hypertension, renovascular disease, and diabetes mellitus. Finally, patients at risk for atheroembolic renal disease usually have multiple risk factors for atherosclerosis and almost always have symptomatic atherosclerosis. ${ }^{1-6,11-14}$ In our series of 52 patients with atheroembolic renal failure, at the time of the diagnosis, all but 1 patient had one or more of the following risk factors and/or prior medical problems: hypertension $(88 \%)$, diabetes mellitus (19\%), tobacco use $(100 \%)$, coronary artery disease $(67 \%)$, peripheral vascular disease $(56 \%)$, cerebrovascular disease $(31 \%)$, and aortic aneurysm $(31 \%)$. A history of chronic renal failure was documented in 29 patients $(56 \%)$. Renal artery stenosis, diagnosed by duplex sonography and/or renal angiography, was documented in 12 patients (23\%).

\section{Renal Involvement}

The kidney is a frequent target organ for cholesterol crystal embolization. Although a small nonsignificant cholesterol crystal embolism can occur in kidneys and be clinically silent, renal clinical manifestations are found at presentation or during the course of the clinical illness in approximately $50 \%$ of the patients. ${ }^{1-3}$ In patients with the disease occurring after angiographic and vascular surgical procedures, the interval from the inciting event to the onset of renal symptoms may vary greatly. Some patients have immediate clinical features, but in others, the onset can be more insidious, with a delay of weeks or months between the precipitating event and clinical features. ${ }^{1-6,11-14}$ In 17 patients who developed atheroembolization after an arteriographic procedure, Frock et $\mathrm{al}^{36}$ found the mean interval between the inciting event and diagnosis of atheroembolic renal disease to be 5.3 weeks.

Cholesterol crystal emboli may give rise to a spectrum of degrees of renal functional impairment. Some patients show only a moderate loss of renal function; in others, severe renal failure requiring dialysis ensues. ${ }^{1-6}$ Clinically, three types of atheroembolic renal disease have been described. In the first type, renal impairment may be abrupt and have a sudden onset. This acute form of renal failure, usually associated with evidence of cholesterol crystal embolization elsewhere, develops a few days after the inciting event and is considered to be the consequence of a massive embolization.

The second type of atheroembolic renal disease, the most frequently observed, is characterized by a subacute time course, probably explained by the foreign-body reaction or the cyclic occurrence of cholesterol crystal emboli showers. In this setting, renal impairment occurs in a 
stepwise fashion. Usually, there is an increase in creatinine level a few weeks rather than a few days after the procedure, and there may be a plateau phase before a further increase in creatinine level occurs. In some patients, it is difficult to determine the exact onset of renal impairment because they are already azotemic when they first come under observation. ${ }^{13}$

In addition to these acute and subacute scenarios, usually after triggering events, a third clinical form, renal atheroembolism, has been observed. It presents as chronic and stable renal impairment associated with the clinical presentation of nephroangiosclerosis and/or ischemic nephropathy. This asymptomatic form, frequently spontaneous, may be missed if renal biopsy is not performed; sometimes, even histological examination may be unconclusive because cholesterol crystal embolization is a patchy process. In this setting, the true role of cholesterol crystal embolism in the progression of renal failure is difficult to assess because they usually cocluster with angiosclerotic and atherosclerotic renal lesions. ${ }^{37,38}$ The renal outcome for patients with atheroembolic renal disease may be variable; some patients deteriorate or remain on dialysis, some improve, and some remain with chronic impairment. In both the acute and subacute scenarios, dialysis may be required in $28 \%$ to $61 \%$ of the patients. ${ }^{2,3,11-13}$ In early reports, the renal outcome was described as quite dismal, with progression over weeks to months to end-stage renal failure. ${ }^{2,3}$ However, more recent experiences suggest less inexorable deterioration, with a possibility for spontaneous recovery of renal function in approximately one third of the patients, even after variable periods of dialytic support. ${ }^{11-13}$ The recovery of renal function is probably caused by several factors, such as reversal of inflammation, resolution of acute tubular necrosis in ischemic areas, and hypertrophy in surviving nephrons.

In our series, the three major clinical forms of renal atheroembolic disease were found. In 18 patients (35\%), the mode of presentation of renal disease was sudden and acute renal failure that occurred 3 to 7 days after the inciting event (angiography). In 29 patients (56\%), a subacute, prolonged, and insidious clinical course was observed, and renal function deteriorated in a step- wise fashion over 2 to 6 weeks. Five patients $(9 \%)$ with a spontaneous form of the disease had chronic and stable renal impairment at time of diagnosis. A spectrum of severity of renal impairment was observed. Uremic signs and symptoms requiring dialytic therapy occurred in 18 patients (35\%); 10 patients were treated by hemodialysis and 8 patients, by peritoneal dialysis. Thirteen patients remained on maintenance dialysis therapy. Five patients ( 3 patients treated by peritoneal dialysis; 2 patients, hemodialysis) recovered sufficient renal function to stop dialysis over an average period of 8 months. In 4 of these patients, dialysis was restarted after few months to control volume overload and/or uremia. At the end of follow-up, only 1 patient was on conservative management without dialysis. In 34 patients $(65 \%)$, the decline in renal function was limited and followed a conservative course. In 32 of these patients, renal function slowly improved or stabilized; in 2 patients, a progressive deterioration of renal function reaching end-stage renal failure requiring dialysis was observed during follow-up.

Renal atheroembolization is often associated with accelerated, poorly controlled hypertension. ${ }^{1-6}$ In a review by Lye et $\mathrm{al},{ }^{3} 48 \%$ of 129 reported patients had severe, accelerated, and/or labile hypertension. Malignant hypertension has also been described..$^{39}$ Potentiation of the reninangiotensin system is the suggested mechanism for hypertension, in which repeated showers of cholesterol crystals are believed to obstruct the renal arterioles, leading to renal ischemia. In our series, a history of antecedent hypertension, exacerbated by the cholesterol crystal embolic disease, was present in 46 patients (88\%); in 6 patients (12\%), hypertension appeared de novo after renal atheroembolization. Finally, frank renal infarction with flank pain or gross hematuria is an unusual clinical presentation of renal atheroembolization, and it has rarely been described. ${ }^{1,6}$

\section{Cutaneous Manifestations}

Among extrarenal findings, the most common is the skin. Cutaneous features most often found are purple toes and livedo reticularis. Typically, the purple/blue toe syndrome presents as the sudden appearance of a small, cool, cyanotic, and painful area of the foot (usually a toe). The 
lesion is tender to touch, usually bilateral, and may progress to ulceration, distal digital infarcts, and gangrene. In the majority of patients, the presence of digital cyanosis is characterized by well-preserved peripheral pulses. ${ }^{1-6}$ Livedo reticularis is also a frequent skin lesion. This consists of a blue-red mottling of the skin in a netlike pattern, most likely caused by obstruction of small arteries, capillaries, or venules in the deep dermis. It is often seen on the feet, legs, buttocks, and lumbar region. Other dermatologic manifestations include nodules, which appear as the result of an inflammatory reaction surrounding cholesterol crystals, purpura, and petechiae. ${ }^{1-3}$

In a review, Falanga et $\mathrm{l}^{40}$ found that cutaneous manifestations were the most common signs of systemic cholesterol crystal embolization and occurred in approximately $35 \%$ of the patients. Livedo reticularis was the most common skin lesion, followed by gangrene, cyanosis, ulceration, nodules, and purpura. In the four largest reports, cutaneous manifestations occurred in $35 \%$ to $90 \%$ of the patients with cholesterol crystal embolization. ${ }^{2,3,11,12}$ The greater incidence of cutaneous lesions reported in recent studies could be explained by close monitoring of the patients during the time course of the disease.

In our series, cutaneous lesions, which usually occurred before renal symptoms, occurred in 96\% of 52 patients. The most frequent skin lesions were cyanosis of the toes $(79 \%)$, livedo reticularis of the lower limbs and abdomen (38\%), and gangrene of the extremities (15\%); two patients also showed ulcerations of the scrotum and penis. The two patients without cutaneous lesions had a spontaneous form of renal atheroembolization presenting as chronic and stable renal impairment.

\section{Gastrointestinal Involvement}

Atheroemboli to the gastrointestinal tract are not uncommon, representing an important and often unsuspected cause of abdominal manifestations. Involvement of the digestive system varies from $18.6 \%$ to $48 \%$, depending on the series cited, and may occur at any site along its length, producing many, often misleading, presentations. ${ }^{41}$ Usually, the prognosis of patients with gastrointestinal disease is poor; the overall death rate is high. Thurlbeck and Castleman ${ }^{15}$ first observed atheroemboli in the surgically resected stomach of an 80-year-old woman. However, they attributed little significance to this finding.

The most common mode of presentation of cholesterol crystal embolism to the bowel is hemorrhage and abdominal pain. ${ }^{1-3,42-44}$ Gastrointestinal bleeding most often results from superficial mucosal ulcerations, erosions, or mucosal infarcts. Clinical presentation may range from occult blood loss and melena to bloody diarrhea, depending on the site and extent of the lesions. Abdominal pain may be caused by noninfarctive ischemia, also inducing malabsorption and diarrhea, or by fibrous stricture with bowel obstruction caused by tissue-repair reactions after repeated showers of atheroemboli. Less frequently, intestinal infarction and perforation involving both the large and small bowel have been described. Cholesterol clefts may be found in angiodysplastic lesions, suggesting an etiologic relationship between them caused by ischemic degenerative changes of the vessel wall. Pseudopolyp formations resulting from tissue-repair reaction have also been described. Pancreatitis is a rare presentation, although the pancreas is a frequent site of emboli. ${ }^{1-3,41,44}$ Clinically, overt hepatitis is rare, and it is characterized by focal liver cell necrosis downstream of lodged arteriolar cholesterol crystals, with an increase in transaminase levels. ${ }^{44}$ Acalculous necrotizing cholecystitis has been reported rarely as a complication of atheroembolism. ${ }^{42-44}$

Intestinal symptoms, consisting mostly of nausea, emesis, abdominal pain, diarrhea, and gastrointestinal bleeding, were present in $10 \%$ to $33 \%$ of the patients in the largest patient series pub-

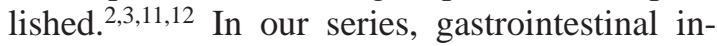
volvement manifested as abdominal pain, diarrhea, and gastrointestinal bleeding in $16 \%$ of 52 patients. Fiberoptic endoscopy and biopsy documented ischemic colitis in 4 patients and gastric bleeding in 3 patients at the site of atheroembolism. One patient had atheroembolic intestinal infarction.

\section{Nervous System Manifestations}

Cerebral cholesterol crystal embolism may be associated with various symptoms, such as transient ischemic attacks, cerebral infarction, amaurosis fugax, paralysis, confusional states, and gradual deterioration of neurological func- 
tion. ${ }^{1-3,45-47}$ In the largest case series, neurological manifestations occurred in $4 \%$ to $23 \%$ of the patients with renal cholesterol emboli syn-

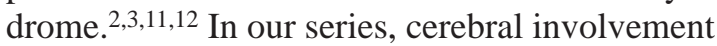
that included transient ischemic attacks, strokes, and changes in level of consciousness was found in $8 \%$ of 52 patients.

However, in clinical studies, a definite diagnosis of atheroemboli in the brain is difficult to make, and it ultimately depends on evidence of cholesterol crystal embolization to other organs. To evaluate the role of cholesterol crystal emboli in the development of cerebral ischemic events, autopsy-proven cases need to be analyzed. Masuda et $\mathrm{al}^{48}$ described 15 autopsy cases with cerebral atheroemboli, identifying two major forms of cerebral cholesterol crystal emboli. The first was characterized by single or multiple cortical infarcts corresponding to the border zones between two main cerebral arterial territories; in this setting, atheroemboli occluded the leptomeningeal arteries with diameters less than $300 \mu \mathrm{m}$. The second form consisted of arterial territorial infarcts located in the territories irrigated by one of the major cerebral arteries or their branches; this occurred when clusters of cholesterol crystal, forming larger emboli, tended to occlude larger arteries. Involvement of the spinal cord artery, which can lead to lower-extremity paralysis, is considered rare. ${ }^{49}$ However, subclinical involvement of the spinal cord artery may be greater. In an autopsy series, Slavin et al ${ }^{50}$ documented cholesterol crystal emboli in spinal and sacral cord arteries in $43 \%$ of 28 patients with evidence of visceral atherosclerotic disease.

Finally, the retina is a frequent target organ of atheroembolism, providing a unique opportunity to observe cholesterol crystal emboli. ${ }^{51}$ Although carotid artery disease more frequently may be the origin of retinal cholesterol crystal emboli, when central nervous system emboli accompany the more generalized form of the disorder, cholesterol crystal emboli arise from the proximal part of the aorta. ${ }^{1-3}$ The incidence of retinal cholesterol crystal emboli, responsible for retinal ischemic events, varies greatly, ranging from $6 \%$ to $25 \%$ of the patients, and depends on the proportion of patients in whom the fundi were examined and the thoroughness of the examination..$^{2,3,11,12}$ In a recent series from a university medical center, ${ }^{14}$ none of the 11 patients with renal atheroembolism had been specifically evaluated for this finding, suggesting that the omission of this noninvasive and diagnostically useful examination is still frequent. In our series, all 52 patients underwent dilated funduscopic examination: classic retinal deposits were documented in 4 patients $(8 \%)$.

\section{Other Extrarenal Manifestations}

Showers of cholesterol crystals may occur in virtually any organ. Myositis and splenic infarcts are some other rare manifestations of cholesterol crystal embolism. ${ }^{1-3,41,52}$ Although uncommon, atheroembolism may involve the coronary arteries; the usual source is the aortic root or the proximal coronary artery. ${ }^{53}$ Recently, cholesterol crystal embolization to the lung has been described, suggesting that atheroembolic renal disease should be considered a new cause of pulmonary-renal syndrome. ${ }^{54}$ Subclinical involvement of the testes, prostate, thyroid, and adrenals has been reported in autopsy studies. ${ }^{1-3,16,30-32}$ Nonspecific findings, such as fever, weight loss, myalgias, and headache, have been reported in a minority of patients and may suggest a systemic disease..$^{1-3,11-13}$

\section{LABORATORY FEATURES}

Laboratory features are nonspecific in patients with cholesterol crystal emboli. However, various laboratory tests help establish the diagnosis of cholesterol crystal embolism. Renal atheroembolic disease is first characterized by elevations in serum creatinine and blood urea nitrogen levels. The variable clinical forms of renal involvement (acute, subacute, and chronic) have been mentioned earlier. Urinary investigation is generally nondiagnostic because it shows such nonspecific alterations as mild proteinuria and microhematuria with hyaline and granular casts. Hematuria is found in $33 \%$ to $40 \%$ of the patients; proteinuria in the nonnephrotic range may be detected in $50 \%$ to $60 \%$ of the patients. $2,3,11,12$ A minority of patients may show nephrotic-range proteinuria, usually in the absence of overt nephrotic syndrome..$^{55,56}$ Accelerated hypertension, a common feature of atheroembolic renal disease, might explain the nephrotic-range proteinuria; alternatively, ischemic glomerular injury, leading to an increased glomerular membrane 
permeability and focal glomerular sclerosis, could be the major factor in the development of heavy proteinuria.

In our series, urinalysis showed mild to moderate proteinuria in the majority of patients. The urinary findings were also unimpressive for vasculitis, frequently associated with a nephritictype sediment. Nephrotic-range proteinuria was documented in three patients: two patients had diabetes and one patient had a previous diagnosis of immunoglobulin A nephropathy. This suggests that the presence of a different underlying glomerular disease may represent an additional possibility to explain the nephrotic-range proteinuria.

Among the extrarenal laboratory features, eosinophilia appears to be the most common finding. From a review of the literature, Kasinath et $\mathrm{al}^{57}$ found an $80 \%$ incidence of eosinophilia when adequate white blood cell counts and differentials were reported. Subsequent reports found an incidence of eosinophilia varying from $14 \%$ to $71 \% .^{3,11,12}$ In our series, hypereosinophilia was found in $62 \%$ of 42 tested patients. The lower incidence of eosinophilia reported in some studies could be explained by less close monitoring of white blood cell differential counts during the time course of the disease. Although persistent eosinophilia has been described, probably resulting from periodic showers of atheroemboli, blood eosinophilia is usually transient. Urinary eosinophilia has also been reported in rare cases. ${ }^{58}$ Leukocytosis, thrombocytopenia, and anemia may also accompany eosinophilia. ${ }^{1-3}$

In an experimental model, Cosio et $\mathrm{al}^{59}$ reported decreased complement levels in rats that developed renal deposits of cholesterol crystals after an intravenous bolus of human atherosclerotic material. They also found hypocomplementemia in seven of nine patients with renal atheroemboli. However, the significance of hypocomplementemia remains unclear because it is relatively mild and transient and has not always been confirmed by subsequent reports. Lye et $\mathrm{al}^{3}$ found hypocomplementemia in only $39 \%$ of the patients with renal atheroemboli. Belenfant et $\mathrm{al}^{12}$ observed serum complement levels within or greater than normal range in 46 patients with renal atheroemboli who had their serum comple- ment levels measured. In our series, complement levels were normal in the 25 patients evaluated.

Laboratory markers of acute inflammation, such as erythrocyte sedimentation rate, serum C-reactive protein level, and fibrin level, may be increased in a substantial proportion of cases. However, because of their nonspecificity, they lack diagnostic utility. ${ }^{2,12}$ Autoantibodies to neutrophil cytoplasmic antigens (ANCA) were negative in all 52 patients in our series. Similar results were reported by Belenfant et al, ${ }^{12}$ who found negative ANCA test results in 25 of 26 evaluated patients.

Laboratory abnormalities may also reflect specific organ involvement. Hyperamylasemia is usually caused by pancreatic involvement, an elevated creatinine phosphokinase level suggests muscular involvement (myositis), and an increase in serum aspartate aminotransferase, serum alanine aminotransferase, alkaline phosphatase, and lactate dehydrogenase levels may reflect hepatic involvement. Renal infarction, although rare in the setting of atheroembolization, typically leads to an increase in serum aspartate aminotransferase and lactate dehydrogenase levels. ${ }^{1-3}$ Hypercholesterolemia is a well-known risk factor for atherosclerosis, of which crystal embolization can be considered a direct complication. However, the most important studies of atheroembolism usually have not reported serum cholesterol levels. ${ }^{2,3,11-13}$ Recently, Mayo and Swartz ${ }^{14}$ identified hypercholesterolemia as a substantive risk factor for renal atheroembolization and found that $27 \%$ of their patients had hypercholesterolemia. In our series, $63 \%$ of 38 patients in whom total serum cholesterol levels were reported had values greater than $200 \mathrm{mg} / \mathrm{dL}$.

\section{DIAGNOSIS}

From a diagnostic point of view, cholesterol crystal embolism has challenged physicians for more than a century. This is likely because atheroembolism is ubiquitous, with random and variable distributions in the body. Thus, cholesterol crystal embolism may mimic a variety of disorders, including systemic vasculitis. ${ }^{1-3}$

Antemortem diagnosis of renal cholesterol crystal embolization is difficult and requires a high index of suspicion. Knowledge of the associated risk factors, recognition of its multiple clinical presentation, and its consideration after 
Table 4. Diagnosis of Atheroembolic Renal Disease in the Literature

\begin{tabular}{lcccr}
\hline & \multicolumn{3}{c}{ Reference } \\
\cline { 2 - 5 } & Fine et al $^{2}$ & Lye et al $^{3}$ & Belenfant et al $^{12}$ & Scolari et al $^{*}$ \\
\hline Publication year & 1987 & 1993 & 1999 & 2000 \\
No. of patients & 221 & 129 & 67 & 52 \\
Clinical diagnosis (\%) & - & 9 & $24 \dagger$ & $21 \neq$ \\
Histological diagnosis (\%) & 100 & 91 & 76 & $79 \S$ \\
Biopsy & 7 & 31 & 36 & 8 \\
$\quad$ Kidney (\%) & 8 & 17 & 22 & 52 \\
Skin (\%) & 8 & 21 & 18 & - \\
Muscle (\%) & 2 & - & - & 2 \\
$\quad$ Gl tissue (\%) & 6 & - & & 6 \\
Other (\%) & 69 & 22 & & \\
Autopsy (\%) & & & & \\
\hline
\end{tabular}

Abbreviation: Gl, gastrointestinal.

* Current study.

† In some patients, clinical diagnosis was confirmed by the presence of retinal cholesterol.

$\ddagger$ Retinal cholesterol emboli confirmed the diagnosis in four patients $(8 \%)$.

$\S$ In two patients, histological confirmation was obtained by nephrectomy specimens.

certain vascular invasive procedures may permit premortem diagnosis in a significant number of cases. The typical patient is a white man aged older than 60 years with a baseline history of hypertension, smoking, and arterial disease. ${ }^{1-6,11-14}$ The presence of a triad composed of a precipitating event, acute or subacute renal failure, and peripheral cholesterol crystal embolization strongly suggests the diagnosis. The presence of other complications of atheroembolism, such as gastrointestinal bleeding and neurological involvement, should raise the level of suspicion. Among the laboratory features, helpful clues to the diagnosis include the presence of eosinophilia ${ }^{57}$ and an increase in the markers of acute inflammation, in association with ANCA negativity. ${ }^{1-3,12}$

Histological confirmation has traditionally been considered essential to the antemortem diagnosis of atheroembolic renal disease. ${ }^{1-6}$ The confirmatory diagnosis is made by means of a biopsy of the target organs, including kidneys, skin, and muscle. On occasion, histological confirmation has been made in less likely target organs, such as gastrointestinal tissue (Table 4). Renal biopsy should be considered the most definitive method of diagnosing atheroembolic renal disease. However, during the acute phase of disease, many patients may be too sick to proceed with renal biopsy: in this setting, the use of an invasive procedure may not be justified. Moreover, because cholesterol crystal embolization is a patchy process, a focal lesion can elude the histological examination. $^{8}$

Alternatively, the biopsy of characteristic cutaneous lesions may yield a positive diagnosis in several cases. Skin biopsy is a simple noninvasive procedure that can be easily performed. Falanga et $\mathrm{al}^{40}$ found that histological confirmation of cutaneous atheroemboli was possible in $92 \%$ of 24 patients in whom a skin biopsy specimen was obtained. Random muscle biopsy from the lower extremities may also have a high diagnostic yield. In the early reports dealing with atheroembolism, antemortem diagnosis was frequently based on muscle biopsy. ${ }^{1-6}$ However, sensitivity of the muscle biopsy has never been evaluated; in addition, skin lesions represent a more easily accessible site. For this and for the frequency of the peripheral ischemic changes, skin biopsy specimens should be considered the best sample of choice for histological diagnosis.

In our series, the diagnosis of cholesterol crystal emboli was confirmed histologically in 41 of 52 patients (79\%); it was based on funduscopic examination showing the presence of retinal cholesterol crystal emboli in 4 patients $(8 \%)$ and was made on clinical grounds alone in 7 patients (13\%) presenting with concomitant acute or subacute renal failure, ischemic peripheral changes, and variable temporal association with a wellknown inciting event. Percutaneous renal biopsy was performed in only 4 patients (8\%). On light 
Fig 1. Kidney biopsy specimen showing cholesterol crystal emboli (arrows) occluding the lumen of an arcuate artery. (Silver methenamine stain; original magnification $\times 10$.)
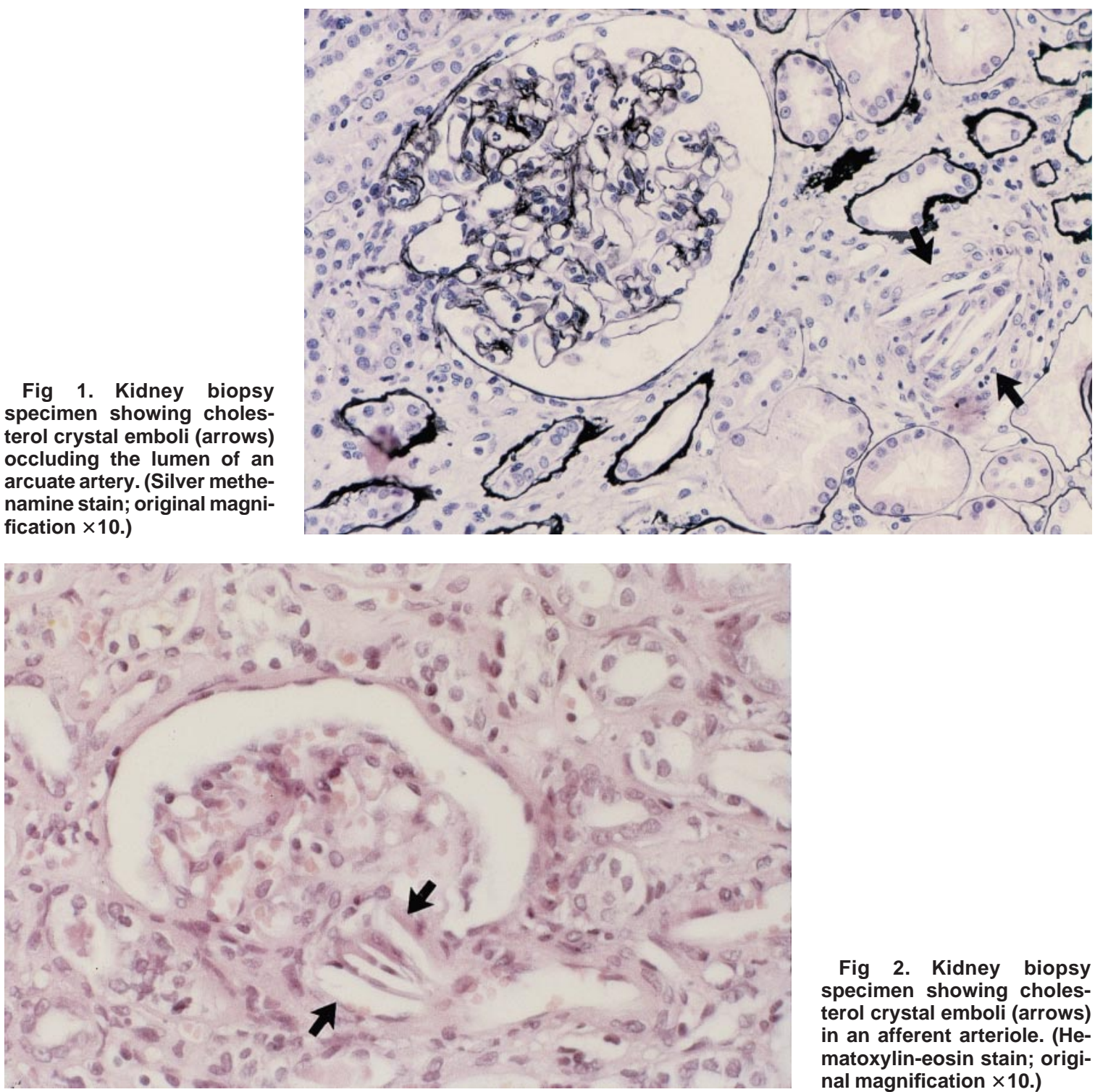

Fig 2. Kidney biopsy specimen showing cholesterol crystal emboli (arrows) in an afferent arteriole. (Hematoxylin-eosin stain; original magnification $\times 10$.)

Fig 3. Kidney biopsy specimen showing cholesterol crystal emboli (arrows) lodging in the glomerular tuft. (Hematoxylin-eosin stain; original magnification $\times 10$.)

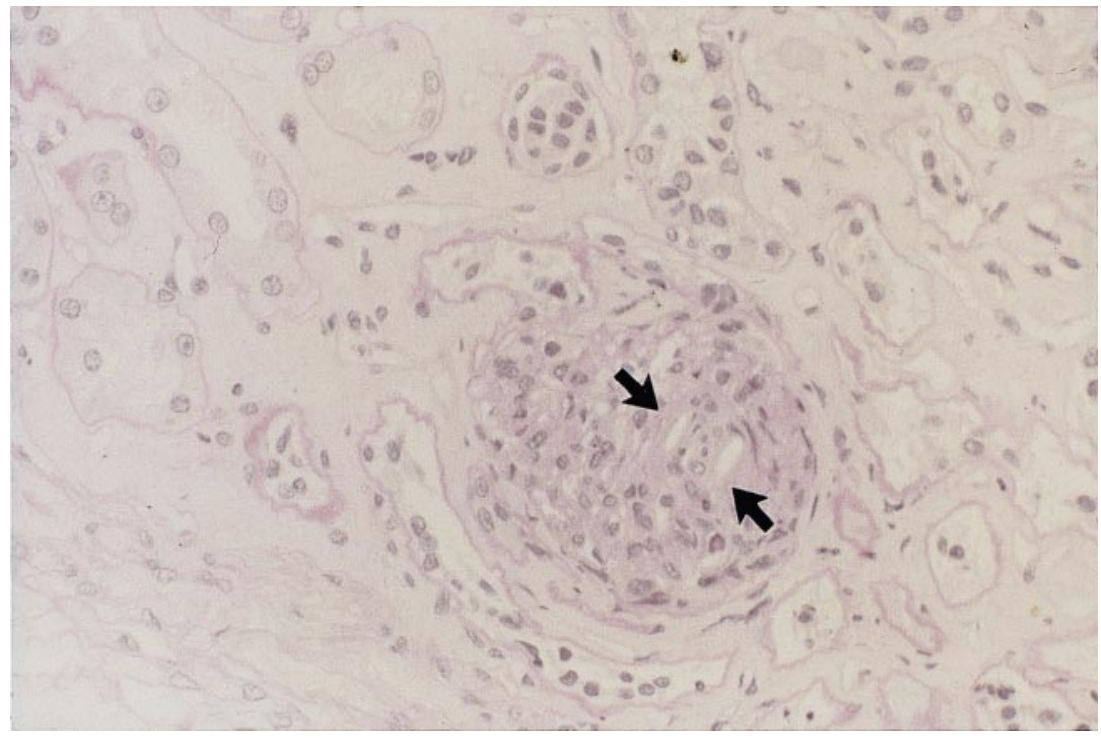




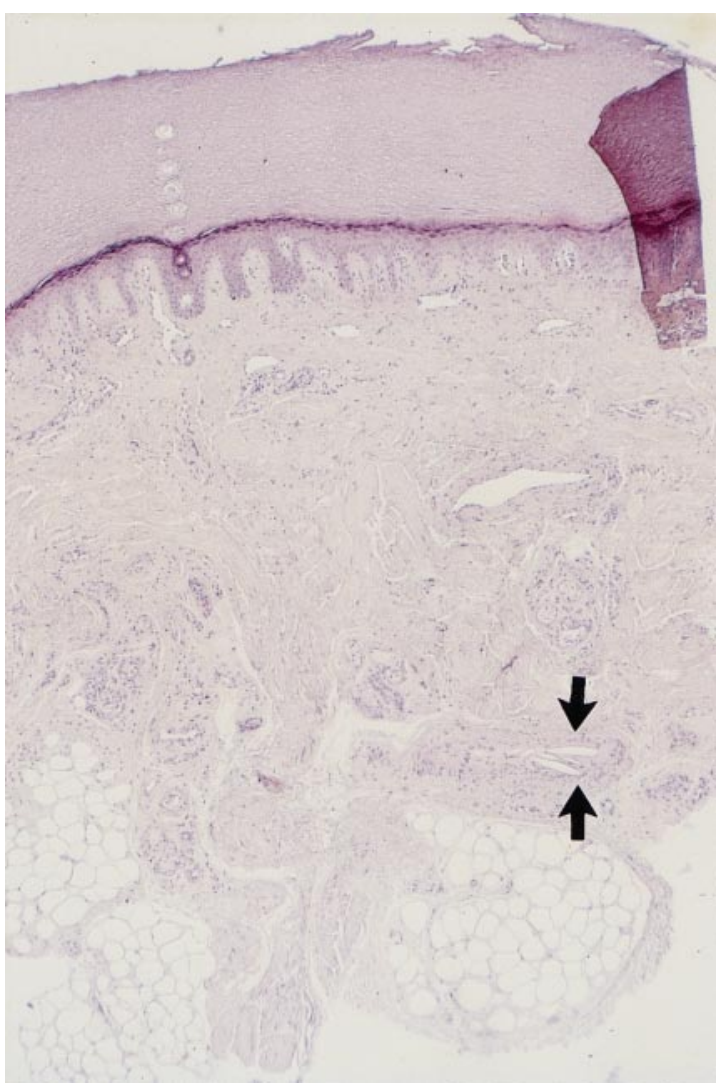

Fig 4. Small artery in the derma occluded by needleshaped clefts (arrows). (Hematoxylin-eosin stain; original magnification $\times 10$.) microscopy, cholesterol crystal emboli consisted of clusters of elongated, biconvex, needleshaped crystals entirely occluding the lumen of small arteries (Figs 1 and 2). In two cases, cholesterol crystals were seen in the glomerular tuft (Fig 3). Varying degrees of arteriosclerotic lesions and hyalinosis and ischemic changes in glomeruli and tubuli from slight to severe were also found. In 1 patient, atheroembolization was superimposed on the classic lesions of diabetic nephropathy. In 2 patients with stable and chronic renal impairment, histological confirmation was obtained by nephrectomy specimens. The indication for nephrectomy was renal carcinoma in both patients. Autopsies were the sole means for histological diagnosis in 3 patients: cholesterol clefts were found in the arterioles of both kidneys. In addition to the kidneys, material from necropsy examination showed cholesterol crystal emboli at different sites: stomach, small bowel, colon, spleen, pancreas, gallbladder, and prostate. Skin biopsy specimens provided the correct diagnosis in 27 patients (52\%), showing cholesterol clefts in the dermis arterioles of the involved areas (Fig 4). In 4 patients (8\%), histological confirmation of cholesterol crystal embolization was made in less likely target organs, such as gastrointestinal tissue (stomach [Fig 5] and colon) obtained on endoscopy. Interestingly, in 2 of these patients, atheroemboli were also found in resected colonic polyps (Fig 6). In 1 patient who presented with an associated hemato-

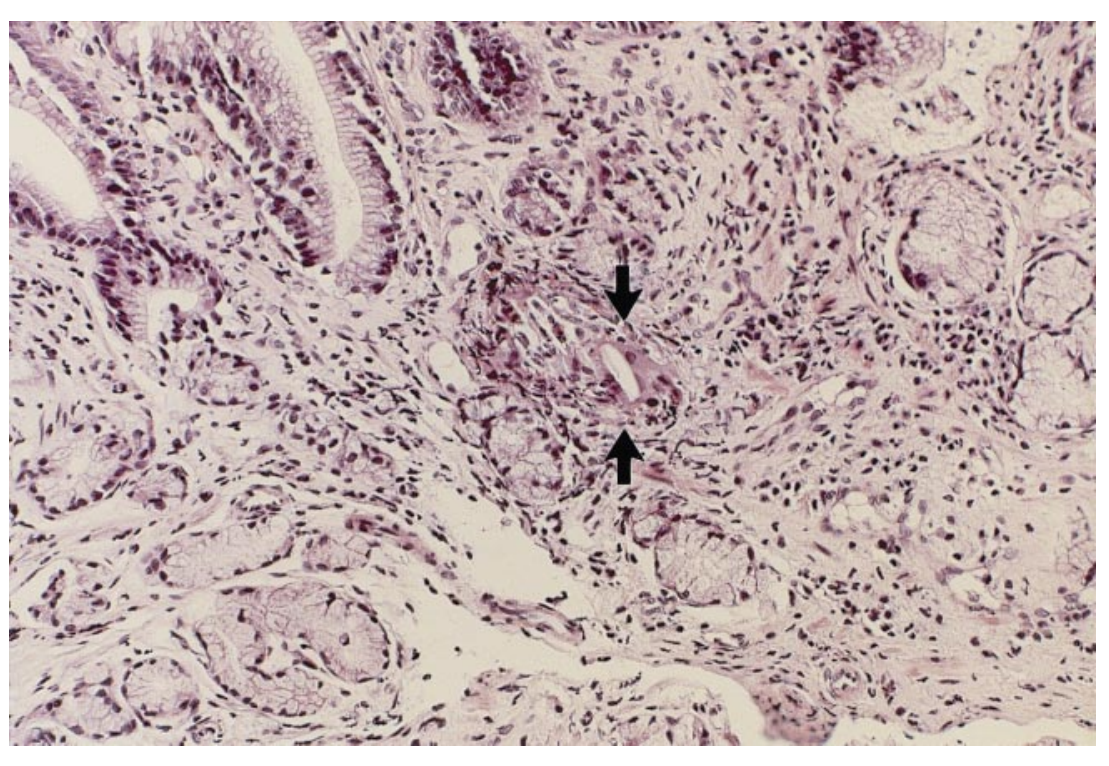

Fig 5. Gastric biopsy specimen showing cholesterol clefts (arrows) surrounded by giant cells in the gastric mucosa. (Hematoxylin-eosin stain; original magnification $\times 10$.) 
Fig 6. Cholesterol crystal emboli (arrows) in a small artery of a colonic polyp. (Hematoxylin-eosin stain; original magnification $\times 5$.)
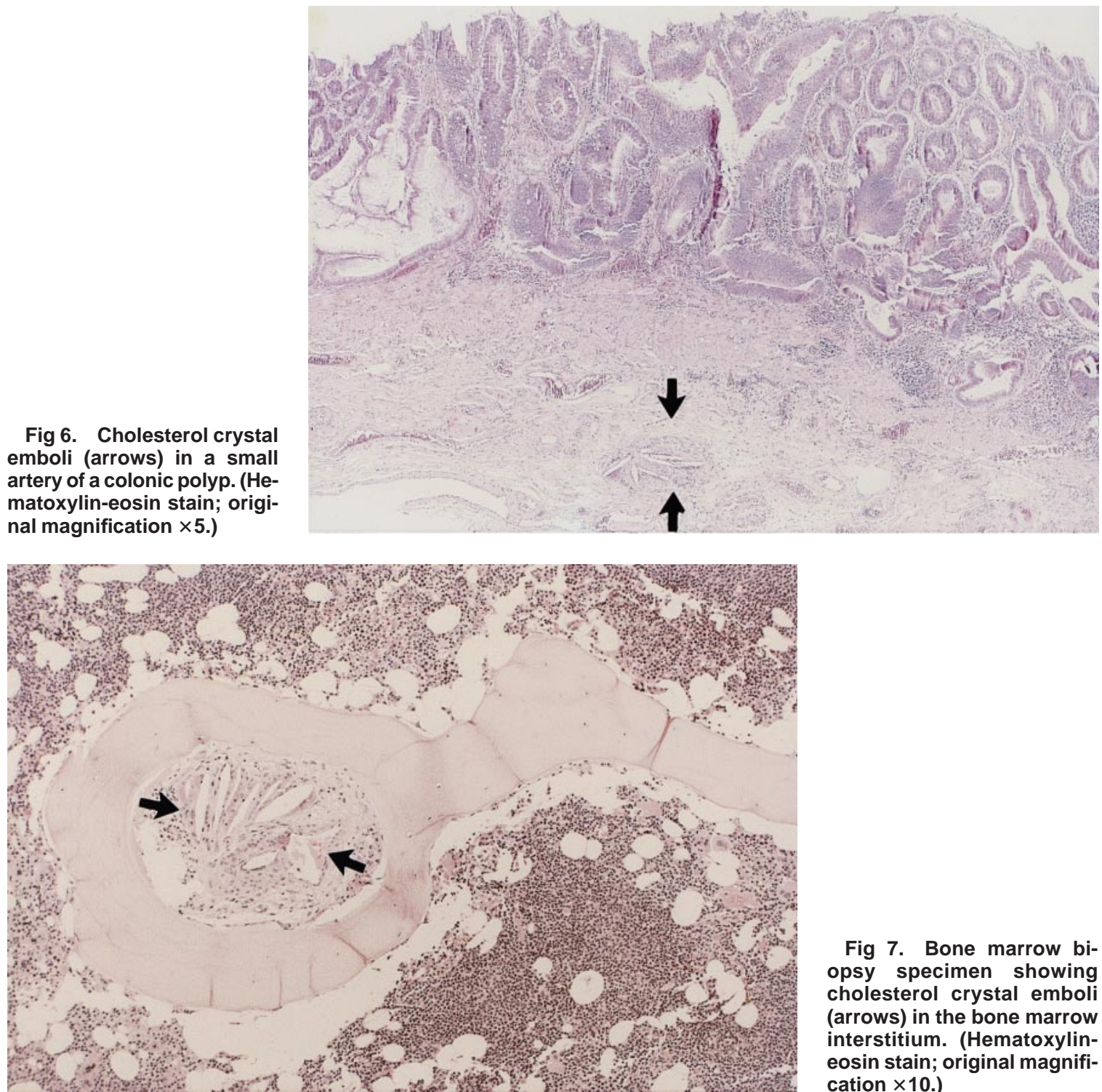

Fig 7. Bone marrow biopsy specimen showing cholesterol crystal emboli (arrows) in the bone marrow interstitium. (Hematoxylineosin stain; original magnification $\times 10$.)

Fig 8. Renal allograft biopsy specimen showing cholesterol crystal emboli (arrows) in an interlobular artery. (Trichromic stain; original magnification $\times 10$.)

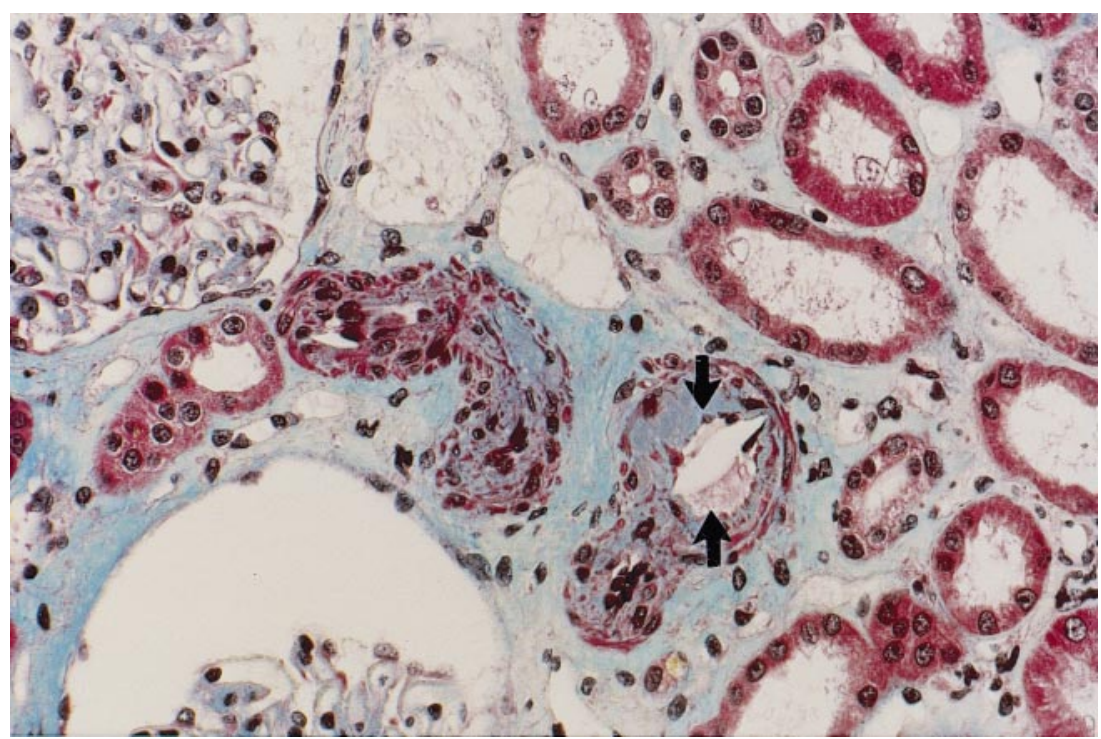


logic disorder, the histological diagnosis was made when the results of an iliac crest bone marrow biopsy were known, showing cholesterol crystal emboli (Fig 7).

Regarding the diagnosis, two final points deserve consideration. First, in the appropriate clinical setting, ophthalmoscopic examination showing retinal cholesterol crystal emboli may establish the diagnosis in a significant number of patients. ${ }^{1-6,11-13}$ Second, in contrast to the accepted teaching, in the presence of a specific set of clinical features (a triad including a precipitating event, subacute renal failure, and peripheral cholesterol crystal embolization), the diagnosis of renal atheroembolic disease may be made without histological evaluation. ${ }^{9,12-14}$

\section{DIFFERENTIAL DIAGNOSIS}

The symptoms and signs of renal cholesterol crystal embolization are nonspecific, diverse, and variable, explaining the notoriety of the disease as the great masquerader. ${ }^{24}$ Thus, a number of clinical entities can simulate cholesterol crystal embolization. The differential diagnosis should include contrast nephropathy, small-vessel vasculitis, and acute interstitial nephritis.

In the immediate phase after an invasive procedure in which dye has been used, differentiation from dye-induced nephropathy is important. Contrast media-associated nephrotoxicity immediately follows the radiographic study. There is an increase in creatinine level a few days after the procedure; peak creatinine level elevation occurs approximately 1 week after exposure and returns to baseline within 10 to 14 days. ${ }^{60}$ Conversely, atheroembolic renal damage frequently has a delayed onset (days to weeks) and a protracted course; the outcome is often poor, resulting in progressive renal failure requiring dialysis. When a fulminant disease develops rapidly after angiography, the concomitant cutaneous, neurological, and gastrointestinal complications usually accompany renal atheroembolic disease. ${ }^{1-6}$

For the presence of eosinophilia, elevated sedimentation rate, weight loss, fever, and multiorgan involvement, atheroembolic disease can masquerade as systemic vasculitis. ${ }^{1-6,24}$ The greatest value in differential diagnosis is the examination of urinary sediment because vasculitis is usually associated with a nephritic-type sediment. ${ }^{1}$ The introduction of the ANCA test, which has $95 \%$ sensitivity for the diagnosis of vasculitis when the disease is active, can help differentiate further the diagnosis of these two clinical entities. ${ }^{12}$ In patients presenting with greater diagnostic confusion, the definitive differentiation of these two entities will rely on histological examination of the involved tissue. The presentation of renal atheroembolism may be indistinguishable from that of an acute tubulointerstitial nephritis, most often induced by drug therapy. ${ }^{4}$ The occurrence of fever, skin rash, eosinophilia or eosinophiluria and a sudden deterioration in renal function in combination with the use of an offending drug is highly suggestive of acute tubulointerstitial nephritis. However, this diagnosis should be frequently confirmed by renal biopsy.

Finally, subacute bacterial endocarditis, which may show neurological, renal, and cutaneous manifestations similar to those of cholesterol crystal emboli, may be a differential diagnosis, especially if hypocomplementemia is present. ${ }^{1-4}$ However, in a patient who harbors occult endocarditis as a source of similar lesions, the correct diagnosis can be suggested by signs and symptoms of systemic sepsis and confirmed by echocardiographic examination and blood culture results.

\section{CHOLESTEROL CRYSTAL EMBOLIC DISEASE IN RENAL ALLOGRAFTS}

Although the clinical features of cholesterol crystal embolism in the native kidney have been well delineated, cholesterol crystal embolization to renal allografts is much less well described. The finding of atheroemboli in the renal allograft was first described in 1985 by Cosio et al, ${ }^{59}$ who found atheroemboli in transplant nephrectomy specimens of a patient who experienced oliguria after cadaveric renal transplantation. Atheroemboli causing injury to the renal allograft may arise from either donor or recipient vessels. When atheroemboli originate from recipient vessels, the clinical presentation is that of a late cholesterol crystal embolic disease, occurring years after transplantation in a stable graft, often associated with the known precipitating factors. Conversely, when the clinical presentation is characterized by early cholesterol crystal emboli associated with initial nonfunction, atheroemboli are believed to arise from the donor's aorta.

Since the first description by Cosio et al, ${ }^{59} 14$ 
Table 5. Cholesterol Emboli in Renal Allografts

\begin{tabular}{|c|c|c|c|c|c|c|}
\hline Reference & $\begin{array}{c}\text { Age R } \\
(y)\end{array}$ & $\begin{array}{c}\text { Age D } \\
(y)\end{array}$ & $\begin{array}{l}\text { Time of } \\
\text { Histological } \\
\text { Evaluation }\end{array}$ & $\begin{array}{l}\text { Emboli } \\
\text { Source }\end{array}$ & Inciting Event & Outcome \\
\hline Pirson et al ${ }^{61}$ & 56 & & $19 y$ & $\mathrm{R}$ & Streptokinase & Functioning graft \\
\hline \multirow[t]{2}{*}{ Fischman et al ${ }^{68}$} & 21 & 45 & 1 wk & $\mathrm{D}$ & Procurement & Nephrectomy \\
\hline & 44 & 65 & 3 wk & $\mathrm{D}$ & Procurement & Nephrectomy \\
\hline \multirow[t]{2}{*}{ Aujla et al62 } & 64 & & $2 y$ & $\mathrm{R}$ & Spontaneous & Functioning graft \\
\hline & 27 & & 1 wk & $\mathrm{D}$ & Procurement & Nephrectomy \\
\hline Jennings et al ${ }^{63}$ & 46 & & $7 y$ & $\mathrm{R}$ & Trauma & Functioning graft \\
\hline Bellamy et al67 & 38 & 59 & At Tx & $\mathrm{D}$ & Procurement & Nephrectomy \\
\hline Singh et al 66 & 40 & 54 & 1 wk & D & Procurement & Functioning graft \\
\hline \multirow[t]{2}{*}{ Bolander and Carter 65} & 59 & 63 & $1 \mathrm{wk}$ & $\mathrm{D}$ & Angiography or procurement & Nephrectomy \\
\hline & 43 & 63 & 3 wk & $\mathrm{D}$ & Angiography or procurement & Nephrectomy \\
\hline \multirow[t]{4}{*}{ De Takats et al64 } & 58 & 66 & At Tx & $\mathrm{D}$ & Procurement & Functioning graft \\
\hline & 50 & 55 & 1 mon & D & Procurement & Nephrectomy \\
\hline & 48 & & $9 y$ & $\mathrm{R}$ & Anticoagulation & Functioning graft \\
\hline & 45 & & $15 y$ & $\mathrm{R}$ & Streptokinase or angiography & Functioning graft \\
\hline Scolari et al* & 48 & 62 & $2 w k$ & $\mathrm{D}$ & Procurement & Functioning graft \\
\hline
\end{tabular}

Abbreviations: Tx, transplantation; D, donor; R, recipient.

${ }^{*}$ Current study.

detailed cases of biopsy-proven cholesterol crystal emboli to renal allografts have been reported in the literature ${ }^{61-68}$ (Table 5). Cholesterol crystals originated from the recipient native vascular tree in five patients who manifested a late cholesterol crystal embolization with a favorable clinical outcome characterized by recovery of renal function. In nine patients, atheroemboli to the allograft were believed to have originated from the donor aorta; in this setting, atheroemboli usually are believed to occur during the workup of a donor, such as coronary arteriography in a multiorgan donor, or during the procurement process. Rarely, cholesterol crystal emboli may occur to the donor kidneys before harvesting, during the donor's life. This group with early cholesterol crystal emboli usually does not show extrarenal manifestations of atheroemboli and has a poor prognosis of the graft: six of nine patients experienced primary allograft nonfunction requiring nephrectomy, and an additional patient required nephrectomy for the concomitant presence of acute rejection. Only two patients with initial nonfunction had spontaneous recovery of renal allograft function. In one of these patients, a contributive role of cyclosporine nephrotoxicity to the patient's clinical course was hypothesized.

Recently, we observed a 48-year-old cadaveric renal transplant recipient who developed renal allograft atheroembolization early posttransplantation. The donor was a 62-year-old man who experienced brain death after an intracerebral hemorrhage; his past medical history was significant for hypertension and tobacco use. At time of procurement, the donor was found to have atherosclerotic aortic plaques. The immunosuppressive regimen consisted of tacrolimus and low-dose steroids. Because of initial nonfunction, a biopsy was performed postoperative day 10. The biopsy findings were consistent with acute tubular necrosis. No sign of acute rejection was found. However, one interlobular artery showed characteristic cholesterol clefts (Fig 8). No extrarenal manifestation was found, and ophthalmoscopic evaluation did not show retinal cholesterol crystal emboli. Abdominal ultrasound did not show aortic atherosclerosis. Hemodialysis, performed without anticoagulation, was required for 2 weeks, then satisfactory renal function was gradually established. The source of the cholesterol crystal emboli in our patient was probably the donor, and the cholesterol crystal embolization likely occurred at the time of harvesting. In contrast to the majority of cases of early cholesterol crystal emboli, our patient had a spontaneous recovery of renal function, confirming that atheroemboli at the time of transplantation may be associated with potential graft viability. 
However, cholesterol crystal emboli could have had a contributory role in the ischemic mechanism leading to acute tubular necrosis, a clinicopathological picture that recognizes a multifactorial pathogenesis. Additional cases of atheroemboli to renal allografts need to be reported to delineate the true natural history of the disease. Because the current trend is to accept older donors and recipients with more advanced atherosclerotic disease, this entity will be recognized more frequently in the future. Thus, cholesterol crystal emboli syndrome must be considered part of the differential diagnosis of primary renal allograft failure. Finally, care must be taken not only at the time of organ procurement, but also during the evaluation of organ donors, to reduce the risk for atheroemboli.

\section{MANAGEMENT AND OUTCOME}

The aim of treatment should be to halt the progression of tissue ischemia and prevent repeated showers of renal cholesterol crystal embolism. At present, no effective treatment is available for this condition. No controlled studies show that medical treatment helps after atheroembolism has occurred. Anticoagulants should be avoided because they may potentiate the problem. ${ }^{1-6,11-13}$ A number of antiplatelet drugs have been tried without success. ${ }^{1,2,37}$ Anecdotal case reports have suggested that pentoxifylline may be beneficial ${ }^{69}$; however, this has yet to be proved. Disagreement exists about steroid treatment. A recent report suggests that steroid therapy might be useful. ${ }^{12}$ However, in previous studies, the use of corticosteroids was associated with $100 \%$ mortality. ${ }^{2}$ Again, it is extremely important to realize that these are all retrospective analyses, with several limitations. Thus, no treatment has been convincingly shown to be effective in altering the course of the disease.

Because the disease is not rare, treatment options are urgently needed. Deterioration in renal function over several weeks suggests an ongoing cholesterol crystal embolic process. Thus, strategies should be developed to stabilize ulcerated or fractured atherosclerotic plaques of the aorta showering cholesterol crystal emboli into renal circulation. Whether lipid-lowering agents are helpful remains uncertain because only occasional cases of atheroembolism have responded to lovastatin and simvastatin..$^{70,71}$ However, in the context of the emerging evidence of statin-induced plaque stabilization and regression, ${ }^{72,73}$ the possible role of an aggressive lipidlowering therapy in the conservative treatment of cholesterol crystal embolization should be evaluated in a proper prospective study. Medical treatment is mostly symptomatic, and supportive measures, including dialysis, are appropriate. Both peritoneal dialysis and hemodialysis have been shown to be adequate means of management of these patients. ${ }^{2,3,11-14}$ Although a particular treatment option is not contraindicated, some investigators suggest that peritoneal dialysis, which avoids anticoagulation, could be the preferred treatment modality. ${ }^{74}$ Conversely, peritoneal dialysis should be excluded in the presence of severe intestinal ischemia and malnutrition. ${ }^{12}$

Surgical treatment could be the definitive treatment, but it is often not feasible and is associated with a high death rate. ${ }^{1-6}$ No randomized trial supports the effectiveness of preventing future atheroembolism by surgical repair or removal of the cholesterol crystal embolic source. Surgery is rarely indicated because the source of cholesterol crystals embolization frequently is not certain. Although we can image the aortic atherosclerotic lesions through a variety of modalities, we cannot predict which lesions will embolize cholesterol crystals. Moreover, patients are usually too weak for a major surgical intervention, and the necessary aortic clamping during surgery would induce a major risk for recurrence.

In a large prospective series, Keen et al ${ }^{75}$ studied 100 patients with atheroembolic disease, of whom only $11 \%$ had renal involvement and the rest had only peripheral symptoms. Occlusive aortic iliac disease and small aortic aneurysm were the most common sources for atheroemboli. Correction of the cholesterol crystal embolic source was achieved with aortic bypass, aortoiliac endarterectomy and patch, femoral or popliteal endarterectomy and patch, and upperextremity bypass. The survival rate was $89 \%$ at 1 year. All seven early deaths occurred in patients with major suprarenal aortic disease, six of whom were undergoing hemodialysis. The investigators suggested that the source of the cholesterol crystal emboli can be eliminated surgically with low mortality or limb loss only when the emboli 
focus is located in the infrarenal aorta. When the suprarenal aorta is involved, greater mortality rates are observed, and they appear to be related to the risk for visceral and renal atheroemboli. Thus, surgical intervention should be limited to life-threatening situations, when immediate survival is at stake. ${ }^{12}$ Similarly, invasive vascular radiological procedures should be precluded or postponed. Prevention is important. The main recommendation is to restrict the indications of angiography and surgical procedures as much as possible in severely atherosclerotic patients. ${ }^{12,37}$ The availability of new noninvasive diagnostic tools, such as spiral computed tomographic angiography, angiomagnetic resonance, and duplex ultrasonography, may partially obviate the risk for catheter-induced embolization.

Patients with atheroemboli have a dismal outlook. In four different reports, a 1-year mortality rate ranging from $64 \%$ to $87 \%$ was noted. ${ }^{2,3,6,11}$ Causes of death were multifactorial, cardiac, ruptured aortic aneurysm, central nervous system, and gastrointestinal ischemia. ${ }^{1-6,11-14}$ However, in a recent study, an aggressive supportive treatment was associated with a 1-year survival rate of $79 \% .{ }^{12}$ This aggressive management was characterized by the avoidance of anticoagulation, good control of hypertension and heart failure, dialytic therapy, and adequate nutrition; finally, aortic manipulating procedures were usually postponed. In our series of 52 patients, the general therapeutic approach was similar. Anticoagulant therapy was discontinued. Further invasive procedures were proscribed. The need for dialysis was evaluated to control both the uremic state and hypervolemia. Target blood pressure was 140/80 mm Hg. During the acute phase, adequate nutritional support was provided by parenteral nutrition in 4 patients; 3 patients required an amputation of a portion of the lower extremity; and 7 patients were treated with pentoxifylline without altering the course of disease. Steroids were not used. The 1- and 2-year survival rates, estimated using Kaplan-Meier actuarial curves, were $69 \%$ and $61 \%$, respectively. Eighteen patients died; 5 patients died during the acute phase of the disease, and 8 additional patients died within the first year of follow-up. In agreement with previous reports, death was most commonly from cardiac causes, gastrointestinal ischemia, and stroke. Together, these data and those of the recent study by Belenfant et $\mathrm{al}^{12}$ suggest that an aggressive therapeutic approach with patient-tailored supportive measures may be associated with a favorable clinical outcome.

\section{ACKNOWLEDGMENT}

The authors thank Fabio Facchetti (Chair of Pathology, University of Brescia) for supplying the photographs for this manuscript.

\section{REFERENCES}

1. Kassirer J: Atheroembolic renal disease. N Engl J Med 280:812-818, 1969

2. Fine MJ, Kapoor W, Falanga V: Cholesterol crystal embolization: A review of 221 cases in the English literature. Angiology 42:769-784, 1987

3. Lye WC, Cheah JS, Sinniah R: Renal cholesterol embolic disease. Case report and review of the literature. Am J Nephrol 13:489-493, 1993

4. Saleem S, Lakkis FG, Martínez-Maldonado M: Atheroembolic renal disease. Semin Nephrol 16:309-318, 1996

5. Colt HG, Begg RJ, Saporito JS, Cooper WM, Shapiro LS: Cholesterol emboli after cardiac catheterization: Eight cases and review of the literature. Medicine 67:389-400, 1988

6. Dahlberg P, Frecentese D, Cogbill T: Cholesterol embolism: Experience with 22 histologically proven cases. Surgery 105:737-746, 1989

7. Panum PL: Experimentelle Beitrage zur Lehre von der Embolie. Virchows Arch 25:308-310, 1862

8. Flory CM: Arterial occlusion produced by emboli from eroded aortic atheromatous plaques. Am J Pathol 21:549565,1945

9. Scoble JE, O'Donnell PJ: Renal atheroembolic disease: The Cinderella of nephrology. Nephrol Dial Transplant 11:1516-1517, 1996

10. Scoble JE: Is nihilism in the treatment of atheroembolic disease at an end? Am J Kidney Dis 33:975-976, 1999

11. Thadani R, Camargo C, Xavier R, Fang L, Bazari H: Atheroembolic renal failure after invasive procedures. Natural history based on 52 biopsy-proven cases. Medicine 74:350-358, 1995

12. Belenfant X, Meyrier A, Jacquot C: Supportive treatment improves survival in multivisceral cholesterol crystal embolism. Am J Kidney Dis 33:840-850, 1999

13. Scolari F, Bracchi M, Valzorio B, Movilli E, Costantino E, Savoldi S, Zorat S, Bonardelli S, Tardanico R, Maiorca R: Cholesterol atheromatous embolism: An increasingly recognized cause of acute renal failure. Nephrol Dial Transplant 11:1607-1612, 1996

14. Mayo RR, Swartz RD: Redefining the incidence of clinically detectable atheroembolism. Am J Med 100:524529, 1996

15. Thurlbeck WM, Castleman B: Atheromatous emboli to the kidneys after aortic surgery. N Engl J Med 257:442447,1957

16. Kealy WF: Atheroembolism. J Clin Pathol 31:984989, 1978 
17. Eliot RS, Kanjuh VI, Edwards JE: Atheromatous embolism. Circulation 30:611-618, 1967

18. Arroyo LH, Lee RT: Mechanism of plaque rupture: Mechanical and biologic interactions. Cardiovasc Res 41:369375, 1999

19. Blankenship JC: Cholesterol embolisation after thrombolytic therapy. Drug Safety 16:78-84, 1996

20. Ramirez G, O'Neill WM, Lambert R, Bloomer A: Cholesterol embolization. A complication of angiography. Arch Intern Med 138:1430-1432, 1978

21. Johnson LW, Esente P, Giambartolomei A, Grant WD, Loin M, Reger MJ, Shaw C, Walford GD: Peripheral vascular complications of coronary angioplasty by the femoral and brachial techniques. Cathet Cardiovasc Diagn 31:165172,1996

22. Gruppo Italiano per lo Studio della Sopravvivenza nell'infarto (GISSI): Effectiveness of intravenous treatment in acute myocardial infarction. Lancet 1:397-401, 1986

23. Blankenship JC, Butler M, Garber A: Prospective assessment of cholesterol embolization in patients with acute myocardial infarction treated with thrombolytic vs conservative therapy. Chest 107:662-668, 1995

24. Lie JT: Cholesterol atheromatous embolism. The great masquerader revisited. Pathol Annu 27:17-50, 1992

25. Jones DB, Iannacone PM: Atheromatous emboli in renal biopsies. An ultrastructural study. Am J Pathol 78:261276,1975

26. Snyder HE, Shapiro JL: A correlative study of atheromatous embolism in human beings and experimental animals. Surgery 49:195-204, 1961

27. Gore I, McCoombs HL, Lindquist RL: Observations on the fate of cholesterol emboli. J Atheroscler Res 4:527535,1964

28. Warren BA, Vales O: The ultrastructure of the stage of atheroembolic occlusion of renal arteries of arterial walls to cholesterol crystals in atheroembolism. Br J Exp Pathol 54:469-475, 1973

29. Saklayen MG: Atheroembolic renal disease. Preferential occurrence in whites only. Am J Nephrol 9:87-88, 1989

30. Cross SS: How common is cholesterol embolism? J Clin Pathol 44:859-861, 1991

31. Moolenar W, Lamers CBHV: Cholesterol crystal embolization in The Netherlands. Arch Intern Med 156:653657,1996

32. Gore I, Collins DP: Spontaneous atheromatous embolization: Review of the literature and report of 16 additional cases. Am J Clin Pathol 33:416-426, 1960

33. Preston RA, Stemmer CL, Materson BJ, Perez-Stable E, Pardo V: Renal biopsy in patients 65 years of age or older. An analysis of the results of 334 biopsies. J Am Geriatr Soc 38:669-674, 1990

34. Stone WJ, Fogo A: Cholesterol embolization, in Martínez-Maldonado M (ed): Hypertension and Renal Disease in the Elderly. Boston, MA, Blackwell, 1992, pp 261-271

35. Drost H, Buis B, Haan D, Hillers JA: Cholesterol embolism is a complication of left heart catheterization. Report of seven cases. Br Heart J 52:339-342, 1984

36. Frock J, Bierman M, Hammeke M, Reyes A: Atheroembolic renal disease: Experience with 22 patients. Nebr Med J 79:317-321, 1994
37. Zuccalà $\mathrm{A}$, Zucchelli $\mathrm{P}$ : A renal disease frequently found postmortem, but rarely diagnosed in vivo. Nephrol Dial Transplant 12:1762-1767, 1997

38. Zucchelli $\mathrm{P}$, Zuccalà $\mathrm{A}$ : Can we accurately diagnose nephrosclerosis. Nephrol Dial Transplant 10:S2-S5, 1995 (suppl 6)

39. Dalakos TG, Streeten DH, Jones D, Obeid A: 'Malignant' hypertension resulting from atheromatous embolization predominantly of one kidney. Am J Med 7:135-138, 1974

40. Falanga V, Fine MJ, Kapoor WN: The cutaneous manifestations of cholesterol crystal embolization. Arch Dermatol 122:1194-1198, 1986

41. Moolenaar W, Lamers C: Cholesterol crystal embolization and the digestive system. Scand J Gastroenterol 26:69-72, 1991

42. Moolenaar W, Lamers C: Cholesterol crystal embolisation to the alimentary tract. Gut 38:196-202, 1996

43. Moolenaar W, Lamers C: Gastrointestinal blood loss due to cholesterol crystal embolization. J Clin Gastroenterol 21:220-223, 1995

44. Moolenaar W, Lamers C: Cholesterol crystal embolization to liver, gallbladder, and pancreas. Dig Dis Sci 41: 1819-1822, 1996

45. Beal MF, Williams R, Richardson EP, Fisher CM: Cholesterol embolism as a cause of transient ischemic attacks and cerebral infarction. Neurology 31:860-865, 1981

46. Prince DL, Harris J: Cholesterol emboli in cerebral arteries as a complication of retrograde aortic perfusion during cardiac surgery. Neurology 20:1209-1214, 1981

47. Laloux P, Brucher JM: Lacunar infarction due to cholesterol emboli. Stroke 22:1440-1444, 1991

48. Masuda J, Yutani C, Ogata J, Kuriyama Y, Yamaguchi $\mathrm{T}$ : Atheromatous embolism in the brain: A clinicopathologic analysis of 15 autopsy cases. Neurology 44:1231-1237, 1994

49. Desnuelle C, Lanteri-Minet M, Hofman P, Butori C, Bedoucha P, Chatel M: Cholesterol emboli with neurologic manifestation in the spinal cord. Rev Neurol 148:715-718, 1992

50. Slavin RE, Gonzales-Vitale JC, Marin OS: Atheromatous emboli to the lumbosacral spinal cord. Stroke 6:411415,1975

51. Hollenhorst R: Significance of bright plaques in the retinal arterioles. JAMA 178:23-29, 1961

52. Robinson R, Pemberton M, Goddard M: Myositis due to cholesterol emboli. Postgrad Med J 69:947-949, 1993

53. Teja K, Crampton RS: Intramural coronary arteritis from cholesterol emboli: A rare cause of unstable angina preceding sudden death. Am Heart J 110:168-170, 1985

54. Sabatine MS, Oelberg DA, Mark EJ, Kanarek DH: Pulmonary cholesterol crystal embolization. Chest 112:16871692, 1997

55. Haqqie SS, Urizar RE, Singh J: Nephrotic-range proteinuria in renal atheroembolic disease: Report of four cases. Am J Kidney Dis 28:493-501, 1996

56. Greenberg A, Bastacky SI, Iqbal A, Borochovitz D, Johnson JP: Focal segmental glomerulosclerosis associated with nephrotic syndrome in cholesterol atheroembolism: Clinicopathologic correlations. Am J Kidney Dis 29:334344,1987 
57. Kasinath BS, Coewin HL, Bidani AK: Eosinophilia in the diagnosis of atheroembolic renal disease. Am J Nephrol 7:173-177, 1987

58. Wilson DM, Salazer TL, Farkouh ME: Eosinophiluria in atheroembolic renal disease. Am J Med 91:186-189, 1991

59. Cosio FG, Zager RA, Sharma HM: Atheroembolic renal disease causes hypocomplementemia. Lancet 2:118121,1985

60. Rudnick MR, Berns JS, Cohen RM, Goldfarb S: Nephrotoxic risks of renal angiography: Contrast-media associated nephrotoxicity and atheroembolism. A critical review. Am J Kidney Dis 24:713-727, 1994

61. Pirson Y, Honhon B, Cosyns JP, van Ypersele C: Cholesterol embolism in a renal graft after treatment with streptokinase. BMJ 296:394-395, 1988

62. Aujla N, Greenberg A, Banner BF, Johnston JR, Tzakis AG: Atheroembolic involvement of renal allografts. Am J Kidney Dis 13:329-332, 1989

63. Jennings WC, Smith J, Cory RJ: Atheromatous embolization as a cause of increasing creatinine levels in a renal transplant patient. Transplant Proc 22:279, 1990

64. De Takats D, Pollock L, O'Donnell P: Is cholesterol embolic disease an unrecognized cause of renal graft dysfunction? Nephrol Dial Transplant 11:1325-1327, 1996

65. Bolander JE, Carter CB: Cholesterol embolization in renal allografts. J Am Soc Nephrol 7:18-22, 1996

66. Singh I, Killen PD, Leichtman AB: Cholesterol emboli presenting as acute allograft dysfunction after renal transplantation. J Am Soc Nephrol 6:165-170, 1995

67. Bellamy C, Paul A, Fleming S: Primary nonfunction of a renal allograft due to atheromatous emboli. Nephrol Dial Transplant 9:182-184, 1994

68. Fischman MA, Hancock W, Bartus SA, Hull D, Schweizer RT: Renal allograft failure from cholesterol emboli. Clin Transplant 3:156-158, 1989

69. Carr ME, Sanders K, Todd W: Pain relief and clinical improvement temporally related to the use of pentoxifylline in a patient with documented cholesterol emboli. A case report. Angiology 45:65-69, 1994

70. Cabili S, Hochman I, Goor Y: Reversal of gangrenous lesions in the blue toe syndrome with lovastatin: A case report. Angiology 44:821-825, 1993

71. Woolfson RG, Lachmann H: Improvement in renal cholesterol emboli syndrome after simvastatin. Lancet 351: 1331-1332, 1998

72. Waters D: Plaque stabilization: A mechanism for the beneficial effect of lipid-lowering therapies in angiographic studies. Prog Cardiovasc Dis 37:107-120, 1994

73. Pitt B, Waters D, Brown WV, van Boven AJ, Schwartz L, Tittle LM, Eisenberg D, Shurzinske L, McCormick LS: Aggressive lipid-lowering therapy compared with angioplasty in stable coronary artery disease. N Engl J Med 341:70-76, 1999

74. Siemons L, Van Den Heuvel P, Parizel G, Buyssens N, De Broe M, Cuykens J: Peritoneal dialysis in acute renal failure due to cholesterol embolism: Two cases of recovery of renal function and extended survival. Clin Nephrol 28:205208, 1987

75. Keen R, McCarthy W, Shireman P, Feinglass J, Pearce W, Durham J, Yao J: Surgical management of atheroembolization. J Vasc Surg 21:773-781, 1995 